Meta

Journal des traducteurs

Translators' Journal

\title{
Procès, procédure, processus
}

\section{Gina Abou Fadel et Henri Awaiss}

Volume 50, numéro 2, avril 2005

Processus et cheminements en traduction et interprétation

Processes and Pathways in Translation and Interpretation

URI : https://id.erudit.org/iderudit/011005ar

DOI : https://doi.org/10.7202/011005ar

Aller au sommaire du numéro

\section{Éditeur(s)}

Les Presses de l'Université de Montréal

\section{ISSN}

0026-0452 (imprimé)

1492-1421 (numérique)

Découvrir la revue

\section{Citer cet article}

Abou Fadel, G. \& Awaiss, H. (2005). Procès, procédure, processus. Meta, 50(2), 620-625. https://doi.org/10.7202/011005ar

\section{Résumé de l'article}

Cette contribution a deux originalités : la première c'est d'être écrite à deux mains, la deuxième c'est d'appliquer une méthodologie de recherche dite " arabe " puisqu'elle se fonde sur la dérivation. Autour de la racine d'un mot s'articule une pléiade de concepts et de termes.

Dans une première partie, nous ferons le procès du système d'enseignement traditionnel par années, en vigueur avant la mise en application de la Déclaration de Bologne ; système qui, malgré tout ce qu'on pourrait lui reprocher, a réussi à former des personnes capables de le remettre en question. La deuxième partie traitera du développement à l'ÉTIB de la procédure mise en place par le Système Européen des Crédits Transférables, plus communément connu sous le sigle ECTS. Le nouveau redéploiement pédagogique fera l'objet de la troisième partie : une répartition en quatre paliers, initiation, stratégies, domaines et synthèse théorique, est venue remplacer l'ancienne partition : traduction générale, traduction spécialisée. 


\title{
Procès, procédure, processus
}

\author{
GINA ABOU FADEL
}

HENRI AWAISS

Université Saint-Joseph, Beyrouth, Liban

hawaiss@usj.edu.lb

\begin{abstract}
RÉSUMÉ
Cette contribution a deux originalités: la première c'est d'être écrite à deux mains, la deuxième c'est d'appliquer une méthodologie de recherche dite «arabe » puisqu'elle se fonde sur la dérivation. Autour de la racine d'un mot s'articule une pléiade de concepts et de termes.

Dans une première partie, nous ferons le procès du système d'enseignement traditionnel par années, en vigueur avant la mise en application de la Déclaration de Bologne; système qui, malgré tout ce qu'on pourrait lui reprocher, a réussi à former des personnes capables de le remettre en question. La deuxième partie traitera du développement à l'ÉTIB de la procédure mise en place par le Système Européen des Crédits Transférables, plus communément connu sous le sigle ECTS. Le nouveau redéploiement pédagogique fera l'objet de la troisième partie: une répartition en quatre paliers, initiation, stratégies, domaines et synthèse théorique, est venue remplacer l'ancienne partition: traduction générale, traduction spécialisée.
\end{abstract}

\begin{abstract}
This article brings about two new innovative features: the first feature lies in the fact that it is co-written, and the second in the fact that it implements a research methodology known as "Arab" since it is based on derivation. A wide variety of concepts and terms revolve around the root of a word. The first part of the study is meant to assess the traditional teaching method applied throughout the years before the implementation of the Bologna Declaration. Despite harsh criticism, this system proved able to form people with the wit to rethink it. The second part will tackle the issue of development of the newly-born process at ÉTIB through the implementation of the European Credit Transfer System, known as ECTS. The newly adopted pedagogical reshuffling will be addressed in the third part of the present study: a four-stage division involving initiation, strategies, fields and theoretical synthesis is bound to replace the old binary partition of general translation $\mathrm{v} / \mathrm{s}$ specialized translation.
\end{abstract}

\section{MOTS-CLÉS/KEYWORDS}

initiation à l'acte de traduire, stratégies de traduction, domaines de traduction, théorie de la traduction, courant traducto-pédagogique

Quand le thème de ce numéro de META «Processus de traduction» nous fut proposé, notre manière de réfléchir à partir des liens de voisinage, voire de cousinage, entre les mots fit surface dans notre esprit. Le mot "processus» appela deux autres mots cousins: procès et procédure. Cette manière d'établir des relations entre les mots est typique de notre culture qui souvent a tendance, pour construire une recherche, à revenir à la racine du mot noyau, considéré comme le tronc de l'arbre généalogique de toute une génération de mots dérivés. De la racine trilitère kataba 
(écrire) dérive toute une famille de mots à connotations différentes, fort utilisés dans la communication quotidienne: kitab (livre), kateb (écrivain), al-kitab (le livre sacré, Coran ou Bible), ahl al kitab (les chrétiens), katatib (écoles primaires), kateb (secrétaire d'état), moukataba (correspondance), maktoub (lettre), maktoub ${ }^{1}$ (ce qui est écrit, le destin, la fatalité), maktab (bureau)...

Quoique appartenant au domaine juridique, les termes de procès, procédure et processus se dotent, dans cette contribution, d'une acception plus large. C'est que les mots ne sont pas figés, ils tolèrent de nouveaux sens sans pour autant perdre leur identité d'origine. Ils feront partie, l'espace de cet article, du domaine traductologique et serviront la réflexion que nous nous proposons d'élaborer ci-après.

\section{J'accuse...}

Les accusations sont en général formulées à l'encontre des personnes. Elles seront ici formulées à l'encontre d'un certain train de vie universitaire qui assurait le transport entre un point de départ et le terminus en quatre années. Celui qui ratait ce train devait attendre le prochain qui ne passait qu'une fois l'an. Celui qui descendait par mégarde dans l'une des stations ne pouvait rejoindre le train suivant mais devait impérativement refaire le trajet à partir du point de départ ou de la station d'avant. Seules quelques lignes de rattrapage pouvaient desservir, en deuxième session, les stations suivantes. Il est vrai que ce train était une sorte d'Orient Express qui assurait tout le confort dont on pouvait rêver, mais seuls pouvaient le prendre ceux qui pouvaient s'en payer le luxe, ceux qui avaient tout le loisir de faire des études et rien que des études. Le trajet était certes long, d'une organisation exemplaire mais monotone, répétitive et les passagers ne pouvaient se déplacer qu'à l'intérieur du même wagon, aucune mobilité vers les autres trains ou les autres parcours n'étaient tolérés. Il arrivait que des passagers, ne pouvant plus supporter la lourdeur de la machine en marche qui ne prenait en considération ni leurs souhaits ni leurs besoins individuels, leur imposant des menus uniques préparés à l'avance, devenaient turbulents et n'arrivaient plus à tenir en place. C'est alors qu'intervenaient les contrôleurs pour les ramener à l'ordre. On lisait parfois même dans les faits divers qu'un passager, étouffé par la rigidité qu'on lui imposait, avait fini par se jeter par la fenêtre. Beaucoup se retrouvaient victimes des calculs d'épiciers car il leur manquait un quart de point pour pouvoir continuer le voyage. Ce malheureux petit quart de point les obligeait alors à descendre du train et à reprendre le trajet à partir de la station précédente.

L'ÉTIB n'a pas échappé à ce train; elle a même péché par excès de zèle. En effet, la moyenne 12/20 était de rigueur, une sorte de passeport obligé pour passer en quatrième année. Intervenait cependant ici la notion de rachat; de longues discussions en jury de délibération tournaient en fin d'année autour de la barre de rachat à adopter. Fallait-il racheter à 11,8 ou à 11,7 ?! Si quelqu'un se hasardait à demander quelle était la différence entre 11,7 et 11,8 ou même 11,5 , des réponses telles que «Il faut absolument maintenir le niveau...», «Il faut être rigoureux et honnête...» fusaient de toutes parts et n'avaient en fait qu'un seul sens : il fallait à tout prix terroriser l'étudiant et maintenir la tension pendant les examens, une sorte d'épée de Damoclès! Pour certains collègues, le 12 n'était qu'une simple transposition de la moyenne de 10. Pour d'autres, c'était la moyenne de 10 majorée d'un plus. Bref, rien n'était tout à fait clair ou objectif et les victimes tombaient par dizaines. Ajoutons à 
ceci l'autonomie de chaque matière par rapport à l'ensemble, chose qui ne permettait aucune compensation, les matières n'étant pas regroupées en modules. Chaque professeur était donc seul maître à bord et faisait, pour ses étudiants, la pluie et le beau temps. De plus, le travail de toute l'année dépendait de l'examen final précédé d'une autre malheureuse notion : la semaine de retraite. N'oublions pas le système de surveillance militarisée où le surveillant croyait que son travail l'obligeait à dénoncer de préférence plusieurs étudiants en flagrant délit. Chez les interprètes, la situation n'était pas meilleure.

Malgré toutes ses bavures, l'«ancien régime» a réussi tout de même à former une élite de traducteurs et d'interprètes qui, bien que conscients des défauts du système, gardent un bon souvenir de leur passage à l'ÉTIB et vont même jusqu'à exprimer leur reconnaissance, fiers qu'ils sont des percées qu'ils ont pu réaliser dans le monde de la recherche et de la profession.

\section{Je me conforme...}

À la suite de la Déclaration de Bologne, l'adoption par plusieurs universités européennes du Système des Crédits Transférables poussa l'Université Saint-Joseph à s'engager dans la même voie. Ce fut pour l'ÉTIB le moment ou jamais de laisser un vent nouveau souffler et emporter les mauvaises herbes. Il n'était nullement question de faire la toilette de l'ancien système mais de l'abolir complètement et de partir sur de nouvelles bases où l'étudiant était roi. Le régime des années fut alors remplacé par celui des semestres, plus léger, moins rigide. Désormais, l'étudiant était placé au cœur de l'opération d'apprentissage, il pouvait avancer à son propre rythme, ne choisissant les matières que pour un nombre de crédits qu'il pouvait assumer ${ }^{2}$. Place était alors faite à l'organisation de sa propre vie, il pouvait par exemple travailler pour subvenir à ses besoins, ralentissant ainsi, dans la mesure tolérée par le règlement, le rythme de ses études.

Le temps d'apprentissage est actuellement subdivisé en cours magistral et en travaux personnels contrôlés (TPC). Le cours appelé «magistral» ne l'est point en vérité; le professeur ne débite pas des informations qui habitent les livres ou sont disponibles sur la toile. Il analyse, synthétise et critique en interaction avec ses étudiants. Son cours est magistral parce que c'est le cours d'un "maître» qui maîtrise parfaitement sa discipline, qui est capable de faire réagir ses étudiants et de les inviter à réfléchir, refusant de leur part toute reproduction de son cours et évitant toute évaluation fondée sur l'apprentissage par cœur des notions. Les TPC, eux, sont la suite naturelle de ce cours. Eux non plus ne sont pas la figure embellie des TP (travaux pratiques) ou des TD (travaux dirigés). Ils sont le temps pendant lequel l'étudiant construit avec son maître son propre apprentissage. Il se prend alors en charge, il n'est plus consommateur des produits de son professeur, mais il est, tout comme lui, artisan de l'enseignement-apprentissage. La relation entre enseignant et étudiant n'est plus celle d'émetteur à récepteur mais plutôt celle d'un couple de danseurs qui accordent leurs pas, attentifs au rythme l'un de l'autre. Il était certes difficile de changer l'ancien pli qu'avaient pris certains collègues - pas tous fort heureusement - mais deux ans après l'application de ce système à l'ÉTIB, l'expérience s'est avérée concluante.

Par ailleurs, à l'instar de tous les groupements qui s'unissent de par le monde, les matières se sont vu regrouper dans des modules où les riches tendent la main aux 
pauvres. Par exemple, dans le module Traduction médiatique, la matière de traduction $\mathrm{B}-\mathrm{A}$ peut racheter la matière $\mathrm{A}-\mathrm{C}$, à condition toutefois que la note de cette dernière n'ait pas été inférieure à 5/20. Si, par malheur, cette note l'était, l'étudiant ne redouble pas le semestre, encore moins l'année, et peut continuer son parcours. Il aura tout le temps jusqu'au L6, le $6^{\text {e }}$ semestre de la licence, pour la réussir.

Mobilité, flexibilité, prise en charge personnelle, maturité, responsabilisation, tels sont les atouts d'un système qui pourrait s'appliquer à toute discipline, sans exception. Certes les réticences ne découlent pas du système lui-même mais sont le fruit de prises de position rigides de la part de ceux qui ont senti à un certain moment qu'on leur ôtait leurs privilèges en tant que "phare du savoir».

\section{Je m’approprie...}

À l'occasion de cette réforme pédagogique et à la suite d'une remise en cause interne que nous avions déjà faite en 1997, un réexamen de nos programmes a été entrepris. À cet effet, une commission formée de responsables et d'enseignants de l'ÉTIB réfléchit sur la structuration du cursus de la traduction sur les cycles de la licence et du master. En recevant les étudiants du secondaire, un besoin se faisait sentir, celui de faire la différence entre la traduction utilisée comme preuve de la connaissance d'une langue étrangère et la traduction enseignée comme acte de communication qui se sert de la langue. Il fallait donc couper le cordon entre la conception traditionnelle de la traduction dite «traduction linguistique» et ce que Jean-René Ladmiral (1998: 155) appelle «la traduction traductionnelle». Un cours fut dédié à cet effet, c'est celui par lequel on accueille les étudiants en L2; il est baptisé Initiation à l'acte de traduire. Il est proposé comme matière disciplinaire à l'ÉTIB et comme matière optionnelle ouverte aux autres étudiants de l'Université, curieux de voir ce qui se passe du côté de la traduction.

Une fois initié, l'apprenti traducteur réalise que tout acte de traduction est avant tout une prise de décisions et que cette dernière devrait se plier à des stratégies qui la gèrent, la menant à bon port. La stratégie, telle que définie dans la Terminologie de la Traduction (Delisle et al., 1999: 77) se fait «en fonction de la visée adoptée pour la traduction d'un texte donné» et "oriente la démarche globale du traducteur à l'égard d'un texte particulier à traduire». Les notion de stratégie(s), de visée, d'adaptation, de traduction idiomatique, de mot à mot, de calque et tous les procédés qui en découlent sont alors inculqués, aussi bien dans leur dimension théorique qu'à travers des applications pratiques, aux étudiants des L3 et L4. Ce cours de Stratégies de la Traduction est fermé au grand public car disciplinaire, il constitue le premier pas vers la spécialisation.

En L5, L6, M1 et M2 intervient une autre notion de base, celle du Domaine de Traduction. En effet, c'est le domaine qui, en quelque sorte oriente le traducteur vers la stratégie à adopter et l'emmène à se pencher, par l'intermédiaire de la terminologie et de l'emploi spécifique de la langue, sur le degré de spécialisation du texte. Mais qu'est-ce qu'un domaine, pourrait-on se demander? C'est avant tout un espace clos à l'intérieur duquel l'homme exerce une activité et une seule. À titre d'exemple, dans le domaine de la navigation spatiale, nulle place n'est accordée à l'opération à cœur ouvert qui relève certes du domaine médical. Le domaine peut se subdiviser en plusieurs sous-domaines : le domaine littéraire accueille la poésie, le roman, le théâtre, la 
bande dessinée... C’est aux spécificités de chaque domaine que sont alors sensibilisés les étudiants capables désormais de jongler avec les stratégies et les procédés de traduction.

Des notions théoriques ont jusque-là été examinées avec les étudiants et le va-etvient entre théorie et pratique a constitué le fondement de l'enseignement. En M3, il est grand temps de reconstituer les courants théoriques de la traduction, de les replacer dans leur contexte historique, d'en tirer les enseignements et de jeter là-dessus un regard critique. Un cours d'histoire de la traduction vient se mettre au service de la Réflexion Théorique. C'est avec ce bagage acquis en L et M que l'étudiant démarre sa première recherche traductologique dans le cadre d'un mémoire qui est axé sur une problématique réflexive bien définie.

Traduction générale, traduction spécialisée, telle était la classification simplifiée selon laquelle nous placions traditionnellement l'apprentissage du processus de traduction. Et d'aucuns de se demander, à juste titre d'ailleurs, qu'est-ce qu'un texte général? Littéraire? Nullement! L'écriture littéraire est des plus spécialisées et des plus complexes. Vulgarisé? Appelons-le donc vulgarisé! Simple? Oui, mais quels sont les critères de la simplicité en écriture? Quant à définir la «traduction générale», voilà une tâche des plus ardues! Comment est-ce qu'une traduction peut être générale? Devant ce questionnement et cette prise de conscience, il était impératif de trouver une progression à l'apprentissage du processus de traduction plus solidement fondée.

Initiation, stratégies, domaines et théorie constituent les paliers de cette progression, paliers communs aussi bien aux étudiants du master professionnel qu'à ceux du master de recherche. En M3, deux bretelles bifurquent du chemin commun, l'une conduit au professionnalisme, l'autre à la recherche et c'est dans le cadre de cette dernière seulement que les cours théoriques sont dispensés et qu'un mémoire est exigé.

\section{Conclusion}

Procès, procédure, processus, trois termes que nous avons voulu rapprocher l'un de l'autre. Malgré leur lien de parenté, notre affinité va vers le processus; c'est que nous ne sommes ni juristes ni juges. Nous appartenons à la traduction, à son enseignement, à la réflexion à laquelle elle ouvre la voie. Nous avons seulement voulu rendre compte de l'expérience que nous avons vécue à l'occasion d'une grande mutation. Notre enseignement pratique de la traduction nous a fourni les éléments de base pour la conceptualisation; des différentes écoles traductologiques et des multiples courants pédagogiques, nous avons réussi à tirer les leçons pour aboutir à une certaine synthèse $e^{3}$ qui nous est propre. C'est ainsi que l'ÉTIB invite à une nouvelle conception de l'enseignement de la traduction; la distribution de la formation en quatre moments : initiation, stratégies, domaines et théories. Morcelés pour les seuls besoins de l'opération pédagogique, ces quatre temps fusionnent en fait ensemble à chaque fois qu'il est question de traduire et se tiennent les coudes pour faire passer le message, porteur de sens. Il est vrai que nous sommes passés, à un certain moment, par le comportement d'élève appliqué qui assimile, reproduit et synthétise mais il est venu le temps de se démarquer. Fort d'une tradition d'échange linguistique dans un pays maritime et d'une expérience universitaire qui s'est confirmée au fil des ans, nous sommes désormais à même de tracer notre propre courant traducto-pédagogique. 


\section{NOTES}

1. Paulo Coelho a baptisé l'un de ses livres Maktoub.

2. Le nouveau règlement des études en vigueur à l'Université Saint-Joseph depuis septembre 2004 stipule, dans son article 12, que: "Dans un cursus, l'étudiant peut avancer à son rythme, en s'inscrivant à des matières pour un nombre de crédits supérieur à 15 par semestre et inférieur à 75 par année académique (pour favoriser le respect des durées minimale et maximale des cursus).» Il est à noter que la licence à laquelle est affectée 180 crédits peut durer de 6 à 12 semestres maximum, le master ( 120 crédits) de 4 à 8 semestres et le doctorat (180 crédits) de 6 à 12 semestres.

3. Toujours ouverte à la réflexion traductologique, l'ÉTIB a depuis 1998 multiplié les colloques internationaux. Elle fête cette année son $25^{\mathrm{e}}$ anniversaire et publie pour l'occasion des ouvrages traductologiques de son propre cru, ainsi qu'une compilation de contributions de traductologues internationaux autour du thème «Pour dissiper le flou» et deux ouvrages de J. Delisle et C. Balliu.

\section{RÉFÉRENCES}

Delisle, J., Lee-Jahnke, H. et M. C. Cormier (1999): Terminologie de la traduction, John Benjamins, Amsterdam.

Ladmiral, J. R. (1998): «Théorie de la traduction: la question du littéralisme», Transversalités 65. 